\title{
Preparation and Characterization of Nonwoven Polypropylene Fabric Irradiation-grafted with Acrylic Acid as Weakly Acidic Cation Exchange Fiber
}

\author{
Li Wei, Junfu Wei, Feng Yuan \& Jing Dong \\ Tianjin Polytechnic University \\ Tianjin Municipal Key Lab of Fiber Modification and Functional Fibers \\ Tianjin 300160, China \\ College of Material Science and Chemical Engineering \\ Tianjin Polytechnic University, Tianjin 300160, China \\ E-mail: wlily721@163.com
}

\begin{abstract}
Graft polymerization of acrylic acid onto nonwoven polypropylene (PP) fabric was carried out by a preirradiation method using a ${ }^{60} \mathrm{Co}$ gamma radiation source. Results showed that $2.2^{\prime}$-Azobisisobutyronitrile (AIBN), its concentration was $0.2 \%$, was very important during the grafting polymerization without removal of polymerization inhibitor of AA. The most favorable grafting polymerization conditions were that the total preirradiation dose was under $60 \mathrm{kGy}$, the monomer concentration was lower than $30 \%$, and the bath ratio was 1:200. FT-IR spectroscopy, surface morphology (SEM), wide angle X-ray diffraction (WAXD) spectra of the original PP and the grafted PP were also studied.
\end{abstract}

Keywords: Polypropylene, Graft, Preirradiation

People have paid more and more attention to the environment protection because of the increasing environmental pollution all over the world. Hence, environmentally clean radiation technologies, such as the radiation induced grafting polymerization, have been an efficient and convenient method for modification of polymeric substrates since the second half of last century. Meanwhile ion exchange fibers have played an more important role in removal of heavy metals from wastewater and acidic gas in recent years, due to their large specific surface areas and high adsorption rates. So the synthesis of these ion exchange fibers by using radiation-induced graft polymerization, have attracted the attention of many investigators (Okamoto, 1984, Yun, L, 1994, et al.). The weakly acidic cation exchange fiber in this study is nonwoven polypropylene (PP) fabric with radiation induce-grafted acrylic acid (AA). PP is a diffusely used material because of its outstanding mechanical properties and cost-effectiveness. This type of ion exchangers with carboxy group is widely used to remove heavy metals from seawater, and ammonia, amines, and alkali aerosols from atmosphere. And the previous research has been mainly focused on the basal conditions of reaction, such as solvent type, comonomer compositions, the total irradiation dose, dose rate and so on, and its applications. Although the preparation of the monofunctional group carboxylate cation exchanger based on strong, chemically inert, and nontoxic fiber has been carried out, the process should been made as perfect and effective as possible.

In generally, grafting polymerization is carried out by three different methods; (I) direct irradiation of fiber in monomer solution (II) vapor phase irradiation method (III) preirradiation. Several procedures for preparing PP fibers with grafted AA are known (U Zhun Zhui, 1963). Tesetlin et al. (Malakhova, L.I., 1972) prepare these fibers by direct irradiation under AA vapor at reduced pressure. This indicates that maintaining reduced pressure of AA in an installation exposed to gamma radiation is a complicated problem. And the problem of efficient removal of the heat of the grafting under these conditions is very complex. Besides, removal of the polymerization inhibitor in AA is a slow process, and the irradiation-grafting polymerization could not be generated when the polymerization inhibitor exists in AA solution. From this standpoint, we discover that a small quantity of 2,2'-Azobisisobutyronitrile (AIBN), which is mixed in the 
aqueous solutions, could get the degree of grafting reach to $50 \%$ upwards and the molecular weight of residual polyacrylic acid in solution could be reached to about 2000-8000, these low molecular weight polymers are traditionally used as dispersants (Das, 2003). The modification of PP by using AIBN in grafting polymerization after preirradiation has seldom been reported. This has stimulated our interest in developing a simply and effective approach to utilize this method for PP modification. And it will be a great improvement in industrialized production of this type of IEF. At the same time, we put a small amount of sulfuric acid into the reactive aqueous solutions on account of the addition of a small amount of sulfuric acid to the reaction mixture resulted in a significant acceleration of the degree of grafting.

The aim of this work is to prepare a cation exchange fiber by $\gamma$ - preirradiation-induced grafting of acylic acid onto nonwoven polypropylene fabric, and evaluate the feasibility for the residual polyacrylic acid in solution as dispersants. The influence of experimental conditions such as the total dose, storage time after preirradiation, the monomer concentration, and the bath ratio on the degree of grafting will be discussed, meanwhile the change of crystallinity of the original PP, the preirradiated PP and the grafted PP will be revealed.

\section{Experimental}

The grafting polymerization of acrycal acid (AA) onto nonwoven polypropylene material was carried out by preirradiation method using a ${ }^{60} \mathrm{Co}$ gamma radiation source at different total radiation dose. The nonwoven PP fabric with density of $22 \mathrm{~g} \mathrm{~cm}^{-2}$ is used as substrate materials produced by China nonwoven Co. Ltd in Xianghe, (Hebei, P.R. China ); Acrylic acid (AA) of purity more than $99.5 \%$ (Tianjin, P.R. China ) was used as a grafting monomer without any further purification, other chemicals were analytic reagent and used as received.

The structure of nonwoven fabric was characterized by VECTOR22 IR-spectrometer (Germany, BRUKER Co.) and its surface was examined by QUANTA200-scanning electron micron microscope (Holand, FEI Co.). Besides, the crystal structure was revealed by D8DISCOVER-WAXD measurements (Germany, BRUKER Co.).

The fabric was preirradiated at room temperature without using additional equipment in irradiation zone. The dose rate was $0.56 \mathrm{Gys}^{-1}$. The total radiation dose ranged from 20 to $100 \mathrm{kGy}$. The polymerization was carried out within 7 days after preirradiation.

The graft polymerization was carried out in aqueous solutions of AA. The AA concentration ranged from 10 to $35 \%$. The bath ratio (the wt ratio of solid and liquid phases) ranged from 1:300 to 1:50. The reactant mixtures in the glass flask were aerated by bubbling nitrogen gas for 5-7 min. Then Mohr's salt ( $\left.2.5 \times 10^{-3} \mathrm{~mol} / \mathrm{L}\right)$ was added to the reaction medium to minimize the homopolyrization of AA during the radiation grafting process. The additive AIBN was always $0.2 \%$. The nonwoven PP fabric after grafted AA was removed in hot distilled water. The products were then dried in a vacuum oven for $8 \mathrm{~h}$ at $333 \mathrm{~K}$ and weighed. The degree of grafting $(\mathrm{G})$ was defined as (Yu. V, 2005):

$$
\mathrm{G}(\%)=\left[\left(w_{g}-w_{0}\right) / w_{0}\right] \times 100 \%
$$

Where $w_{g}$ and $w_{0}$ represent the weights of initial and grafted fibers, respectively.

The influence of various parameters on the grafting copolymerization, such as reaction temperature, additives, preirradiation dose, reaction time, monomer concentration, and bath ratio on the degree of grafting was studied.

Agitate the nonwoven PP ion exchange fabric (degree of grafting: $44 \%$ ) in $50 \mathrm{ml}$ HCI solution $(0.5 \mathrm{~mol} / \mathrm{L}$ ) for 10 minutes, then washed through Buchner funnel with deionized water until the eluate reached neutral. Wash the burette with a little water and poured $25 \mathrm{ml} \mathrm{NaCI}$ solution $(1 \mathrm{~mol} / \mathrm{L})$ into it, some salt became pyrolysis. Titrate the ion exchange fabric with $\mathrm{NaOH}$ solution $(0.1 \mathrm{~mol} / \mathrm{L})$ while agitating. Test the $\mathrm{PH}$ value at regular intervals until it reached 10 with a glass-electroded $\mathrm{PH}$ meter. Draw the titration curves with the volume of $\mathrm{NaOH}$ consumed by per mass unit as the $\mathrm{X}$-axis and the relevant $\mathrm{PH}$ value as the $\mathrm{Y}$-axis.

\section{Results and Discussion}

Grafting was predominantly controlled by both concentration of trapped radicals and monomer diffusibility in the polymer matrix. For the samples irradiated at a dose under $80 \mathrm{kGy}$, grafting was proceeding every day within one week. The reaction was carried out at $343 \mathrm{~K}$, which favored the degradation of hydroperoxides. The composition of irradiated nonwoven PP fabric stored for 30 days approached to that of unirradiated samples (Medyak, 2001). In the study, when the storage time after irradiation exceeded seven days, the degree of grafting tended to zero. This demonstrated that the concentration of free radicals become very little.

It can be seen from Fig.1. That the degree of grafting AA in this curve increased as the absorbed dose increased from 30 $\mathrm{kGy}$ to $80 \mathrm{kGy}$, but it then decreased from $80 \mathrm{kGy}$ to $100 \mathrm{kGy}$, because the degree of grafting, in the preirradiation process, rested with the concentration of peroxides. The degree of grafting reached the peak when the irradiation total dose was $80 \mathrm{kGy}$. Liquid-phase grafting polymerization of AA on nonwoven polypropylene fibric after preliminary gamma irradiation was studied as influenced by the ratio of the concentration peroxides formed during gamma $\left({ }^{60} \mathrm{Co}\right)$ irradiation of polypropylene in the air. Therefore, as the total radiation dose increased, the concentration of peroxide increased and so did the degree of grafting. But the homopolymerization of acrylic acid would prick up if the total 
radiation dose was too big, which was not favorable for the use of ion exchange fiber. So, we usually controlled the total radiation dose under $60 \mathrm{kGy}$.

It can be seen from Fig. 2 that the degree of grafting increased as the monomer concentration increased. When the AA concentration was lower than $30 \%$, the grafting polymerization proceeded satisfactorily with the appropriate degree of grafting. When the AA concentration was greater than, or equal to $50 \%$ in solutions, the homopolymer yield increased along with a sharp rise in the degree of grafting. In this case, it was difficult to wash out gelatinous polyacrylic acid from the grafted nonwoven PP fibric.

As seen from Fig.3, when the bath ratio was from 1:50 to 1:200, the degree of grafting increased, and then it decreased with the continue increasing of the bath ratio. The concentration of acrylic acid monomer in the system and concentration of peroxide in the system may be the two factors that influence on the change of degree of grafting with the bath ratio. When the bath ratio was low, the amount of acrylic acid was the major influence factor on the degree of grafting. As the bath ratio increased, the amount of acrylic acid in solution increased and thus the degree of crafting increased. But when the bath ratio reached a certain value, the degree of grafting became to be determined by the comparative concentration of peroxides in the system instead of the amount of acrylic acid. As the bath ratio continued to increase, the comparative concentration of peroxides decreased which led to decrease of grafting active center concentration and therefore led to decrease of degree of grafting.

Fig.4 showed the IR spectra of the original (curve 1) and the grafted nonwoven PP (curve 2) fabric. There were strong adsorption bands at $1455 \mathrm{~cm}^{-1}$ and $1372 \mathrm{~cm}^{-1}$, corresponding to the bending vibrations of $\mathrm{CH}_{2}$ for both $\mathrm{PP}$ and the grafted PP fabric. Compared to the original fabric spectrum, the appearance of the new bands at $1730 \mathrm{~cm}^{-1}$ and $3500-2900 \mathrm{~cm}^{-1} \mathrm{O}-\mathrm{H}$ was due to the stretching vibrations of $\mathrm{C}=\mathrm{O}$, and at $1470-1410 \mathrm{~cm}^{-1}$ corresponding to the bending vibration of $\mathrm{O}-\mathrm{H}$.

That was an evidence of AA had grafted onto the nonwoven PP fabric after the preirradiation and grafting polymerization.

Fig.5 (a), (b) and (c), (d) compared the original fabric with grafted fabric surface at different amplificatory times. The diameter of fiber increased obviously after grafting polymerization, and the smooth surface of the original PP turned into rough. The branch of polyacrylic acid grafted onto the PP randomly during the irradiation and most of them were vertical to the axis of fiber. A heterogeneous grafting layer liking brush structure were formed on the surface of fiber after grafted, resulting in that the fiber became thickness and coarse. That was an evidence of AA had been grafted onto the PP nonwoven fabric during the reaction.

The theoretical static exchange capacities (SEC) of carboxylate ion exchangers were calculated from the degree of AA grafting to the PP fabric. The theoretical SEC for cations agreed well with the experimental values. The difference between the theoretical and experimental SEC was no higher than $12 \%\left(\mathrm{SEC}^{\mathrm{t}}=4.2\right.$ and $\left.\mathrm{SEC}^{\mathrm{e}}=3.7 \mathrm{mmol} / \mathrm{g}\right)$ at $(44$ wt \%) degree of AA grafting to the PP fabric. This suggested the absence of cross-linking during the grafting and accessibility of all carboxyl groups in the grafted fabric.

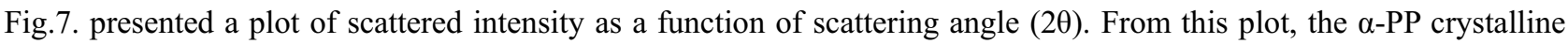
reflections of the original PP can be indexed as the $(110)$ at $2 \theta=14.45$, the $(040)$ at $2 \theta=17.15$, the $(130)$ at $2 \theta=18.80$. The $\alpha$-PP crystalline reflections of the preirradiated PP can be indexed as the $(110)$ at $2 \theta=14.30$, the $(040)$ at $2 \theta=16.75$, the $(130)$ at $2 \theta=18.35$. The $\alpha-P P$ crystalline reflections of the grafted PP can be indexed as the (110) at $2 \theta=14.25$, the (040) at $2 \theta=16.75$, the $(130)$ at $2 \theta=18.40$.

To reveal the impact of preirradiation and grafting modification on the crystal structure or crystallite size of PP, WAXD measurements were performed. The crystallite size $(D)$ vertical to the lattice plane $(h k l)$ could be obtained according to the Scherrer's equation (Alexander LE, 1969):

$$
\mathrm{D}=\mathrm{k} \lambda / \beta \cos \theta
$$

Where $\mathrm{k}$ was the factor of the crystal figure, taking $\mathrm{k}=0.89$. $\lambda$ was the wavelength of the $\mathrm{X}$-ray $(\lambda=1.54056 \AA$, taking $\AA=0.1 \mathrm{~nm}$ ), and $\theta$ was the diffraction angle. $\beta$ was equal to $\left(B^{2}-b_{0}{ }^{2}\right)^{1 / 2}$, where $B$ was the width at half-tallness of the diffraction peak and $b_{0}$ was the broadening factor of the instrument. If we took no account of the lattice distortion, the equation could be simplified as follows:

$$
D=\mathrm{K} \lambda / \beta \cos \theta=0.89 \times 1.54056 \times 57.3 / B \cos \theta
$$

The results of WAXD for the original PP, the preirradiated PP and the grafted PP fabric were shown in Table 1. In Table 1, I was the intensity of the crystalline peaks and $B$ was the peak width at half height, $d_{h k l}$ was the space between lattice planes $(h k l)$.

As shown in Fig.6, the plot of the original PP indicated that the nonwoven polypropelene fabric in this study was melting spinning elicited from boiling pentane. Three curves exhibited scattering angles under these conditions, indicating the $\alpha$-phase monoclinic structure (Guan Y, 2003). The $d$ spacing of the original PP and the preirradited PP 
had a little changed while $D$ increases. But the $d$ spacing of the preirradiated PP and the grafted PP had slightly changed while $D$ decreases. The results can be explained by the heterogeneous nucleation induced by AA. The nucleation accelerated the crystallization in which the growth of grains was restricted for their crushing each other in the course of their growing. The over-dosing of AA onto the backbones of PP tended to increase the crystallite size.

\section{Conclusions}

(1) Preirradiation-induced graft polymerization of AA onto nonwoven polypropelene fabric was carried out effectively by a preirradiation method using a ${ }^{60} \mathrm{Co}$ gamma radiation source at $343 \mathrm{~K}$ with adding $0.2 \%$ of AIBN without removal of polymerization inhibitor of AA.

(2) The most favorable grafting polymerization conditions were that the total preirradiation dose was under $60 \mathrm{kGy}$, the monomer concentration was lower than $30 \%$, and the bath ratio was 1:200.

(3) The FT-IR and SEM of the grafted PP showed that carboxyl group was successfully introduced onto nonwoven polypropelene.

(4) The WAXD spetra of the original PP, the preirradiated PP and the grafted PP indicated that the over-dosing of AA onto the backbones of PP tended to increase the crystallite size.

\section{References}

Alexander LE. (1969). X-ray Diffraction Methods in Polymer Science: New York.

Bondar, Y., Kim, H.J., Lim, Y.J., Kravets, L. (2003). Cation-Exchange Fabric Prepared by Electron Beam-Induced Graft Copolymerization of Binary Monomer Mixture. Macromolecular Symp. 202: 167-178.

Das, Kalyan, K., Somasundaran, P. (2003). Flocculation-Dispersion Characteristics of Alumina Using a Wide Molecular Weight Range of Polyacrylic Acids. Coll. and Surf. A: Physicochemical and Engineering Aspects. 223(1-3): 17-25

Guan Y, Wang SZ, Zheng AN, (2003). Journal of Applied Polymer Science. 88:872.

Gupta, B., Jain, R., Anjum, N., Singh, H. (2006). Preirradiation Grafting of Acrylonitrile onto Polypropylene Monofilament for Biomedical Applications: I .Influence of Synthesis conditions. Radi. Phys. and Chem. 75 (1): 161-167.

Jain, R., Gupta, B., Anjum, N., Revagade, N., Singh, H. (2004). Preparation of Antimicrobial Sutures by Preirradiation Grafting of Acrylonitrile onto Polypropylene Monofilament. II. Mechanical, Physical and Thermal of characteristics. Jour. of Appl. Poly. Scie. 93 (3-5): 1224-1229.

Le Thuaut, P., Martel, B., Crini, G.., Maschke, U., Conqueret, X., Morcellet, M. (2000). Grafting of Cyclodextrins onto Polypropylene Nonwoven Fabrics for the Manufacture of Reactive Filters. I .Synthesis Parameters. Janu. of Appl. Poly. Scie. 77 (10): 2118-2125.

Malakhova, L.I., Vlasov, A.V., Mikhailov, N.V., and Tsetlin, B.L. (1972). Vysokomol Soedin, Ser A. 14(4): 751-755.

Medyak, G. V., Shunkevich, A. A., Polikarpov, A. P., Soldatov, V. S. (2001). Features of Preparation and Properties of FIBAN K-4 Fibrous Sorbents. Russ. Jour. of Appl. Chem., 74(10): 1658-1633.

Okamoto, J., Sugo, T., Katakai, A., Omichi, H. (1984). Complex-forming Polymer Prepared by Electron Beam Radiation-induced graft polymerization. Radi. Phys. and Chem. 25 (1-3): 333-342.

U Zhun Zhui, Stasyuk, Kh.A., Kocherginskaya, L.A., et. al., (1963). Chemisorption Fibers. 5: 12-15.

Wei, J.F., Wang, Z.P., Zhang, J., Wu, Y.Y., Zhang, Z.P., Xiong, C.H.. (2005). The Preparation and the Application of Grafted Polytetrafluoroethylene Fiber as a Cation Exchanger for Adsorption of Heavy Metals. Reactive and Functional polymers, 65(1-2): 127-134.

Yu. V. Bondar', Hong Je Kim, and Yong Jin Lim. (2005). Russian Journal of Applied Chemistry, 78(5): 811-814.

Yuan, S., Wang, Y., Lan, S., Lu, Y., Zeng, H. (1998). Study on the Physical Chemical Properties of FFA-1 Ion Exchange Fiber. Chin. Jour. of Reac. Poly. 7 (2):54-60.

Yun, L., Zhu, Z., Hanmin, Z. (1994). Studies on Preparation and Ion-exchange Properties of Weakly Acidic Cation Exchange Fiber by Preirradiation-induced Graft Copolymerization with Electron Beam. Jour. of Appl. Poly. Scie. 53 (4): 405-410. 
Table 1. Crystal parameters of the original PP, the preirradiated PP and the grafted PP from WAXD

\begin{tabular}{|c|c|c|c|c|c|c|}
\hline sample & $h k l$ & $2 \theta$ & $d_{h k l}(\AA)$ & $\mathrm{I}(\mathrm{cps})$ & $B(\mathrm{o})$ & $D(\AA)$ \\
\hline \multirow[t]{3}{*}{ PP } & 110 & 14.45 & 6.12 & 148 & 1.10 & 71.99 \\
\hline & 040 & 17.15 & 5.16 & 154 & 2.40 & 33.11 \\
\hline & 130 & 18.80 & 4.72 & 123 & 4.70 & 16.94 \\
\hline Preirradiated & 110 & 14.30 & 6.19 & 468 & 0.80 & 98.97 \\
\hline \multirow[t]{2}{*}{ PP } & 040 & 16.75 & 5.29 & 515 & 1.00 & 79.41 \\
\hline & 130 & 18.35 & 4.83 & 288 & 1.60 & 49.74 \\
\hline Grafted & 110 & 14.25 & 6.21 & 645 & 1.10 & 71.98 \\
\hline \multirow[t]{2}{*}{ PP } & 040 & 16.75 & 5.29 & 600 & 1.40 & 56.72 \\
\hline & 130 & 18.40 & 4.82 & 342 & 1.80 & 44.22 \\
\hline
\end{tabular}

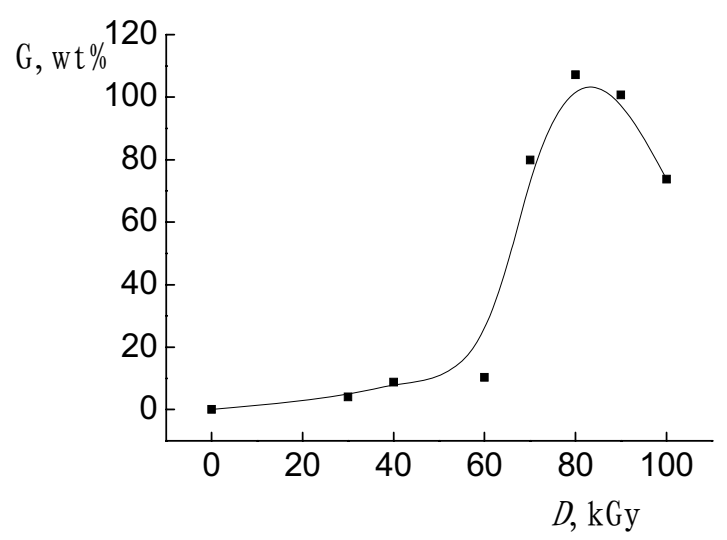

Figure 1. The degree of grafting $G$ vs. the total radiation dose $D$ 


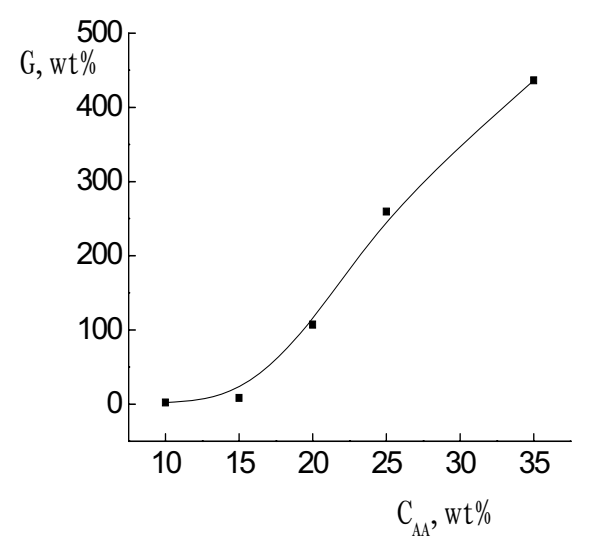

Figure 2. The curve of degree of AA grafting $G v s$. the AA concentration $C_{\mathrm{AA}}$ (polymerization at $348 \mathrm{~K}$ for 2 h)

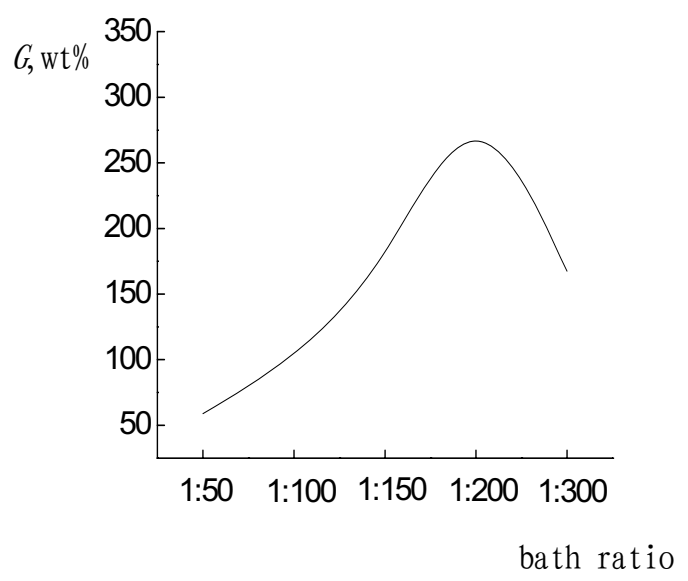

Figure 3. The degree of grafting from a $20 \%$ solution at $348 \mathrm{~K}$ for $2 \mathrm{~h} v$ s. the bath ratio

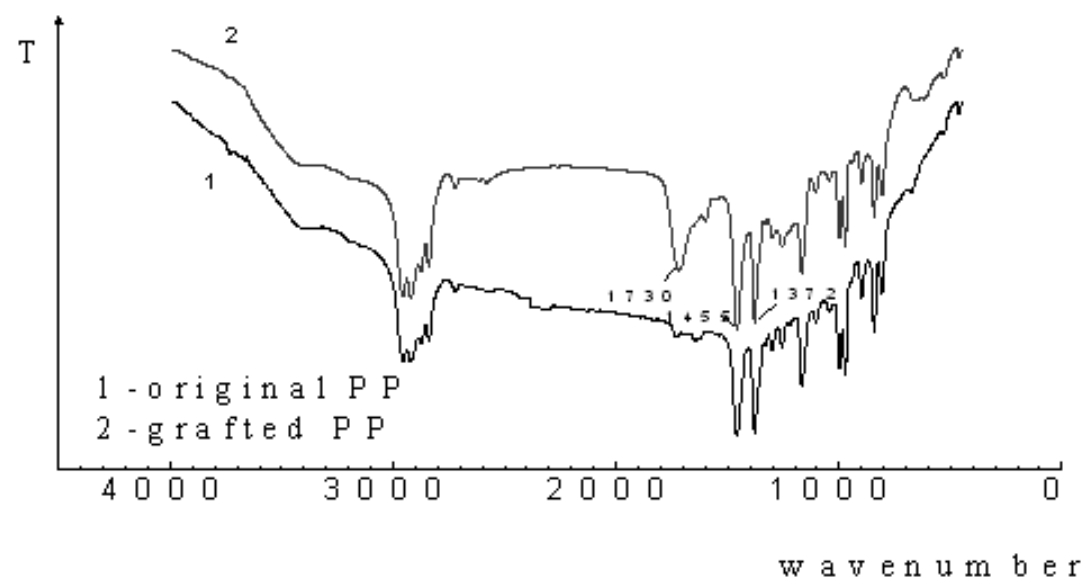

Figure 4. The IR spetra of (1) the original and (2) the grafted PP nonwoven 


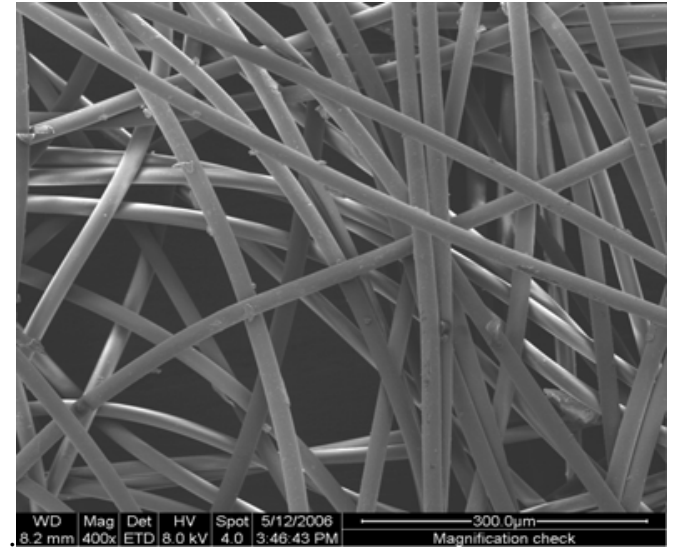

(a)The original PP nonwoven (400 times)

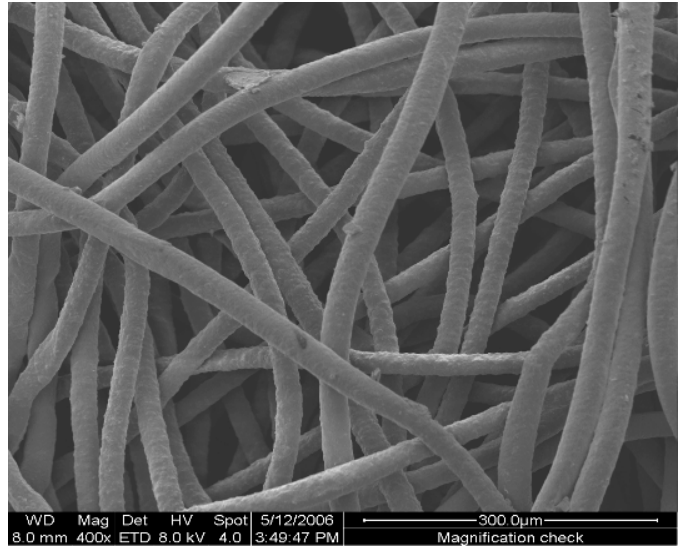

(b) The grafted PP nonwoven (400 times)

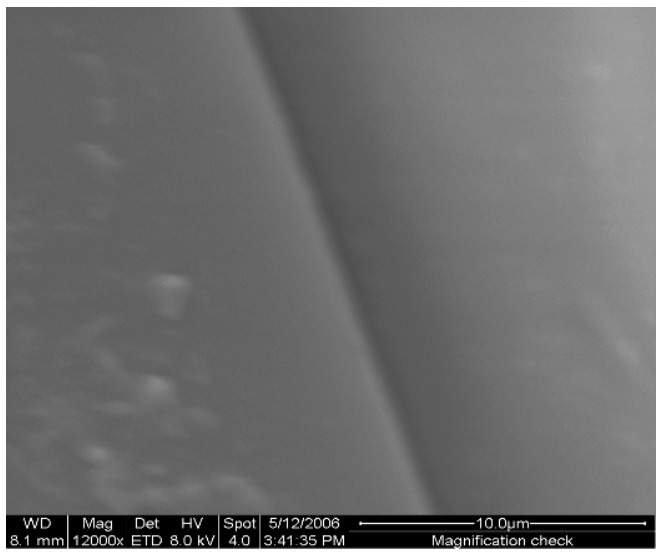

(c) The original PP nonwoven (12000 times)

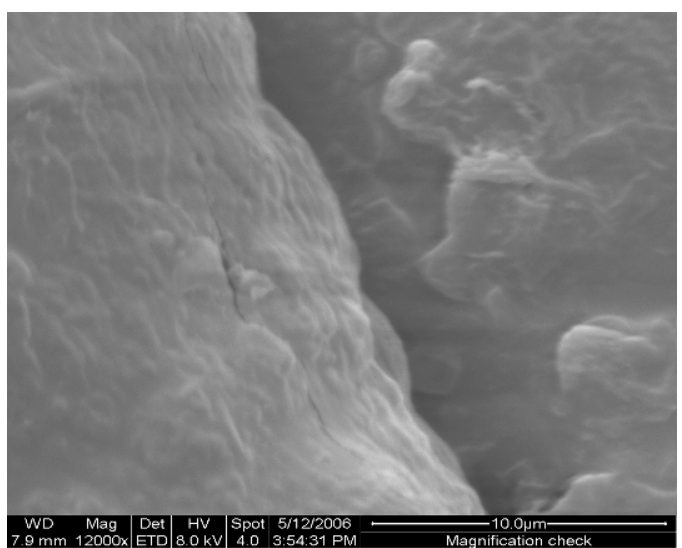

(d) The grafted PP nonwoven (12000 times)

Figure 5. SEM micrograph of the original PP and the grafted PP nonwoven

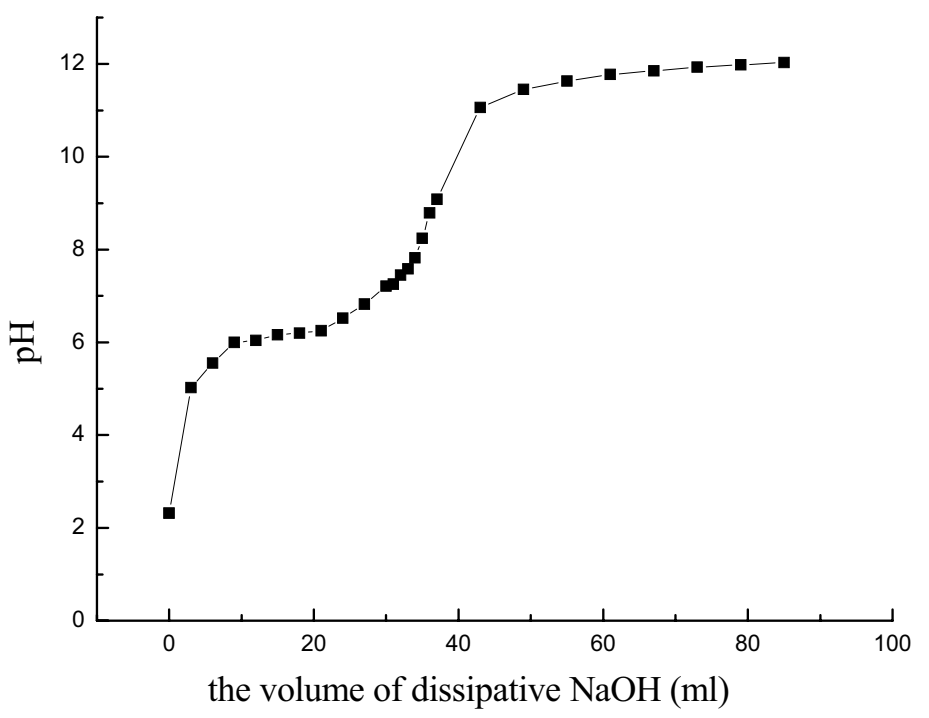

Figure 6. The experimental SEC of 44 wt \% degree of grafting PP fabric 


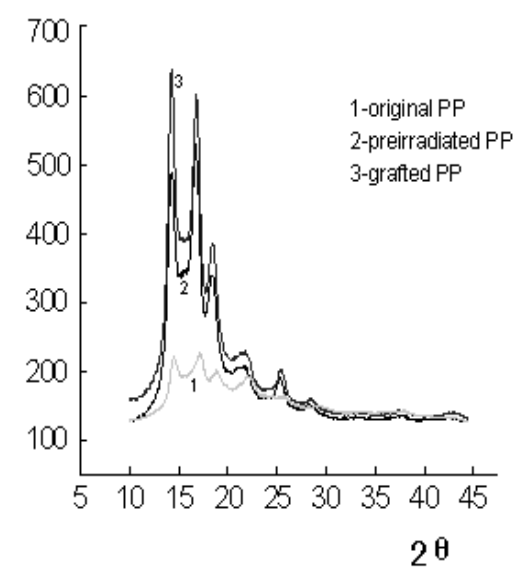

Figure 7. The WAXD spectra of (1) the original, (2) the preirradiated and (3) the grafted nonwoven PP fabric 